\title{
Effective Automatic Fault Detection in Transmission Lines by Hybrid Model of Authorization and Distance Calculation through Impedance Variation
}

\section{Dr. Bindhu V,}

Professor and Head,

Department of Electronics and Communication Engineering,

PPG Institute of Technology,

Villankurichi Saravanampatti P.O,

Coimbatore, India.

\section{Dr. G. Ranganathan,}

Head of the department,

Department of Electronics \& Communication Engineering,

Gnanamani College of Technology,

Namakkal, India.

\begin{abstract}
Fault detection in the transmission is a challenging task when examining the accuracy of the system. This fault can be caused by a man-made force or by using concurrent overvoltage in the power distribution line. This research focuses on two sections to handle the power transmission line problem and can be rectified as previously stated. An intelligent approach is utilized for monitoring and controlling line faults in order to improve the accuracy of the equipment in transmission line fault detection. After several iterations of the procedure, the combination of line and master unit improves the system's accuracy and reliability. The master unit identifies faulty poles in the network based on the variation of current and voltage of each node and calculates the distance between the station and the faulty node to reduce manual effort. In the proposed work, many sensors are used to detect the line fault in a network by placing the appropriate point. The pure information can be transferred to an authorized person or unit after many iterations due to knowledgeable devices. The faulty status of the pole information is displayed in the control unit by a display unit comprised of an alarm unit to alert the corresponding section using ZigBee techniques. The GSM unit provides the faulty status of an
\end{abstract}


Journal of Electronics and Informatics (2021)

Vol.03/ No.01

Pages: $36-48$

https://www.irojournals.com/iroei/

DOI: https://doi.org/10.36548/jei.2021.1.004

authorized person to rectify the problems immediately which further improve the reliability of the system. When compared to existing methods, our hybrid proposed method achieves a higher accuracy of $90 \%$. This method aids to reduce the labor costs gradually to visit all-pole points instead of faulty pole points and thereby increasing the reliability of the electrical consumers.

Keywords: Transmission line faults, Microcontroller, ZigBee, GSM

\section{INTRODUCTION}

Globally, the power supply system includes the generation unit, transmission unit, and distribution unit. These units are carrying out their assigned functions and activities. The monitoring transmission phase is more difficult and expensive everywhere [1]. The power is already being transmitted through the lines that have been built between the nodes in networks. The tower is used to maintain the cables for transmission lines from the substation to many nodes in the area network, such as domestic and commercial points [2]. This power range also is very high and gives to the distribution unit. The different transmission line is connected with many distribution units for customer equipment service [3]. The distributed unit is reducing the power range from high to low and distributes it to appropriate nodes in the network. The power transmission lines consist of many properties for occurring faults between nodes [4]. The electrical faults are happening due to many natural parameters lightening, tree fallen on lines, flying giant bird between the line during a heavy storm. The causes are faults in the transmission line, which should be identified and corrected as soon as possible [5]. The accuracy and reliability maintenance is a very challenging task in transmission line problems. Besides, the electrical equipment short circuit or damaged scenario is unpredictable and impossible to solve in transmission lines. Fault detection is an important consideration to the safeguard of the power line for the distribution unit. Most of the methods are semi-automatic to detect the problems in transmission lines [6]. Figure 1 shows the main functional units for fault detection in the transmission line. 


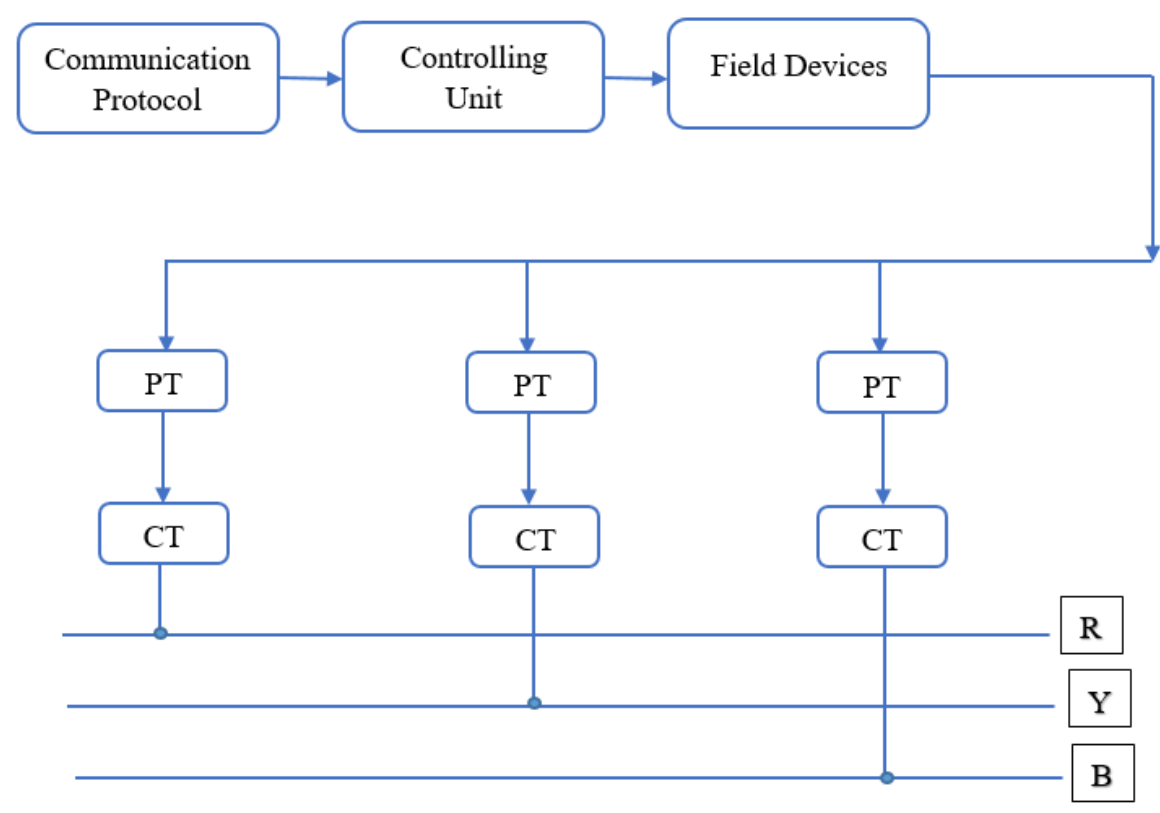

Figure 1 Main units of General Automatic Fault Detection in Transmission Line

This type of identification will be more effective and not satisfying with accuracy and reliability due to many components. Many units are using semi-automatic methods to converts the energy from one to another. So energy consumptions are more and computation time also high in their procedure [7]. There is an absence of effective examination, reliability, and accuracy. The identification of faulty poles in a transmission line leads to sensing the voltage and current output. Most of the research articles fail to provide the distance calculation between sources to faulty poles [8]. This is our motivation and planning to construct the model accordingly. The combination of problem finding and statistical analysis with distance calculation is very rare in modern techniques. Therefore, the proposed model is constructed to solve many problems in transmission lines that exist already. Many investigations including the magnetic field sensor coils is used to detect the fault in the transmission line which is more expensive [9]. During our proposed model, the cost-effectiveness is compared with the existing models. The classification fails in many semi-automatic methods from termination to terminal in the network. An accurate faulty pole location is based on error finding and distance calculation from one to another [10]. These units are non-homogeneous with many trees and loads in the line which makes it complex to find fault. Impedance-based methods are mostly cost-effective for 
Journal of Electronics and Informatics (2021)

Vol.03/ No.01

Pages: $36-48$

https://www.irojournals.com/iroei/

DOI: https://doi.org/10.36548/jei.2021.1.004

terminal measurement. The combination of knowledge-based and impedance measures can be providing good accuracy and reliability [11].

\subsection{Problem statement}

The reduction of response time needed to repair faults in order to achieve optimal transmission line reliability. The cost-effectiveness of the communication protocol provides a reliable product achievement environment. The implementation of the control system in the substation with knowledgeable devices was accurate.

\subsection{Proposed Solution}

This research article comprises efficient impedance and knowledgeable hybrid techniques to identify the fault in the transmission line. The hybrid technique is used to increase productivity and reduce the response time due to its double unit consist of line and master unit control. The separation of the line and the master unit can provide well-reduced computation time and reliability due to the accurate finding of the system.

\subsection{Scope of the research article}

This research comprises the design of an efficient approach for fault identification in transmission lines and includes comparing several existing methods with our proposed hybrid model. This may lead to ensure stability in a good position and increase the accurate fault identification after several iterations.

\section{ORGANIZATION OF THE RESEARCH}

The structure of this article is organized as follows; Section 3 gives recent technology of fault detection of the transmission line. Section 4 describes an efficient method with a block diagram. Section 5 addresses the results and discussion of proposed methods with existing methods. The conclusion and the future task discusses in section 6 . 
Journal of Electronics and Informatics (2021)

Vol.03/ No.01

Pages: $36-48$

https://www.irojournals.com/iroei/

DOI: https://doi.org/10.36548/jei.2021.1.004

\section{PRELIMINARIES}

This section comprises related works about fault detection in the transmission line. There are many types of detection called fully automated fault detection and semi-automated fault detection, manual fault detection in the transmission line. The communication protocol for exchanging the data between poles to the system is necessary before understanding fault detection. The distance calculation is very important to select the appropriate communication protocol for fault detection. This communication protocol is selecting based on bit rate, range, nodes between source to destination, cost, and so on. A. Lavric et al., proposed a ZigBee communication protocol for the transmission fault detection module [12]. M. N. Jivani et al., discussed WiFi importance in GSM-based automation systems [13]. Xiaoxin Xu et al., proposed about wireless healthcare monitoring system by ZigBee technology. Sathesh et al., discussed smart homes with environmental concerns in future challenges through Wi-Fi Technology. This ZigBee technology has a range of around 100 metres, which is greater than other technologies such as Wi-Fi and Bluetooth. During the development of a communication protocol, network topology is an important parameter to consider when choosing a path. It is based on the geographical area over a communication range, structure consideration of local area network, outdoor environment nodes, the total number of nodes calculation in a region [14].

A. A. Siddiqui et al., the author discussed the lighting control system comprises of various topology can be constructed and influenced in local area network such as a tree, star, mesh, bus, and ring topologies. This star topology can have only one master to control all the slaves in their network is a suitable choice of any dominant communication protocol [15]. Other topologies are needed to construct the model with multiple routers and nodes which leads to cost expensive. This configuration setup has been chosen based on the application and network model. Siddiqui et al., proposed the configuration of the group-based control system for the street light control system. They concentrated on preferred costing with total expenses [15]. But this method cannot operate for individual utility pole since group-based control. The ZigBee communication protocol will support individual utility poles well and determine the distance between poles in a fast manner. The ZigBee-based integrated control system is used to analyze the status of the utility pole which should be monitored continuously. Also, it is consuming less number of nodes used between the utility poles. This makes the fastest delivery between a node 
Journal of Electronics and Informatics (2021)

Vol.03/ No.01

Pages: $36-48$

https://www.irojournals.com/iroei/

DOI: https://doi.org/10.36548/jei.2021.1.004

to nodes. As a result, a model is proposed to built with the ZigBee communication protocol to detect faulty signals between utility poles.

\section{METHODOLOGIES}

These parameters are considered in our proposed structure to construct the communication protocol for fault detection between poles.

The proposed system comprises identifying a faulty pole, a faulty lamp in the pole, and the distance between poles. Figure 2 depicts the preliminary work of our proposed system. Field devices are active poles and it's connected with Base Transceiver Station (BTS) which is used to receive and collect the signal from field station called a base station. Base Station Control (BSC) operates based on the activities of the transmission line that can be categorized and provides the switching system for the further classified process. The signal conditioning unit is controlling the incoming signal to better transmission.

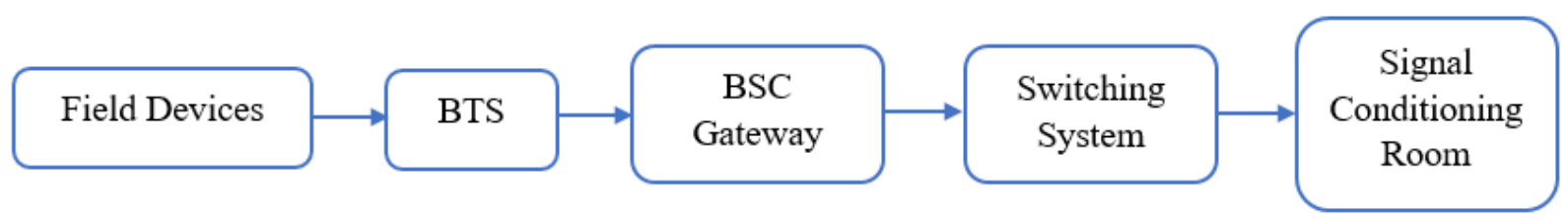

Figure 2 Ground station for transmission control

\subsection{Proposed Detection methods}

\subsubsection{Impedance with frequency factor}

The detection is based on impedance with frequency components of the system. For the impedance-based estimation process, the voltage and current values are measured on both sides of the pole. The fundamental frequency in the line terminal is measured with voltage and current before and during the pole fault. The single-phase ground fault identification with circular properties, identifying a line problem of a short circuit between line and ground through the use of physical contact. Here, the proficient electronic devices are constructed with oscillography function. This function will be installed at the substation level, and information will be taken as reading with all electrical parameters [16]. In general, this procedure faces the problem of 
Journal of Electronics and Informatics (2021)

Vol.03/ No.01

Pages: $36-48$

https://www.irojournals.com/iroei/

DOI: https://doi.org/10.36548/jei.2021.1.004

statistical estimation under reliable conditions. As a result, the specific procedure should be followed in the iterative process to identify the fault type [17].

\subsubsection{High frequency and waveguide factor}

This procedure is functioning with high frequency with a traveling waveguide signal for identifying the fault generation pole. But the implementation of this procedure is very complex in the power network. This reflection-based transmission is providing high accuracy and the cost is very high. Our proposed method can be upgradeable to GSM and ZigBee technologies [18] [19] [20]. The installation of the GPS is comprised of many transient sensors to detect and display software tools. But this fault transient is very big complex to detect in the field. The proposed approach is based on knowledge-oriented such as statistical analysis with microcontroller unit. The probability can be a support to detect the future prediction of the fault that occurs due to circuit break due to high voltage, an abnormal condition of the power line feeders, short circuit due to internal signal conditioning unit [21] [22] [23]. The faulty pole is identified and used to calculate the distance between the source and the destination of the pole. This method is used to achieve an accurate finding with an estimation of the faulted unit. Fault distance measurement at the substation is computed by a combination of estimation and accurate sensor output. Threshold values can be assigned to the default values of poles in a network when they are in normal condition [24].

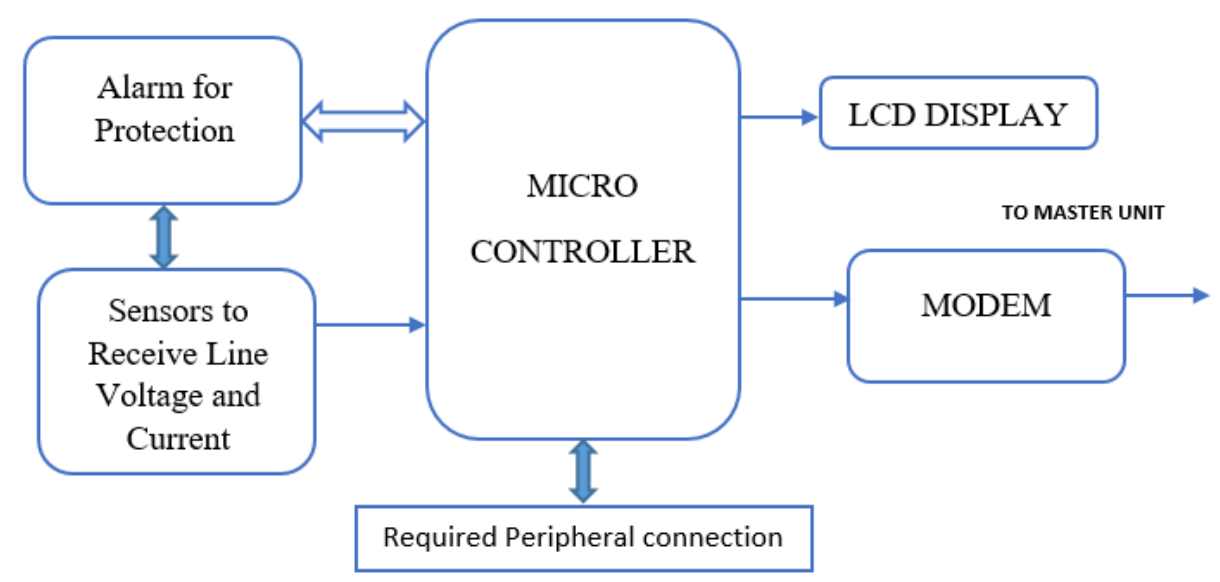

Figure 3 Connected components with microcontroller of Line unit 
Journal of Electronics and Informatics (2021)

Vol.03/ No.01

Pages: $36-48$

https://www.irojournals.com/iroei/

DOI: https://doi.org/10.36548/jei.2021.1.004

The changes in this value can be viewed as faulty poles in terms of voltage and current checking with the estimation of fault points in the network. If both the procedure is identified as same $100 \%$ it is a fault point in a network. And the calculation between the station to the pole in a network takes place by node addition procedure. To transmit data from the microcontroller unit in the line unit, the intelligent device is linked to a display unit, sensor module model, and modem. The proposed systems have three major components: a communication protocol, a knowledgeable controlling unit, and an input module, as shown in Figure 3. The CT and PT transformers are connected to the $\mathrm{R}, \mathrm{Y}$, and $\mathrm{B}$ which receive the power signal using the protecting unit of the proposed module. Furthermore, the signal can be converted into a digital format for analysis by a intelligent device known as a microcontroller. The heart of our proposed module is a microcontroller, which serves as the controlling component of the proposed approach. The programming portion is also written in the EEPROM for our convenience. A microcontroller device can perform the classification, which is used to find faulty poles based on the threshold value and distance calculation between the source and the faulty pole.

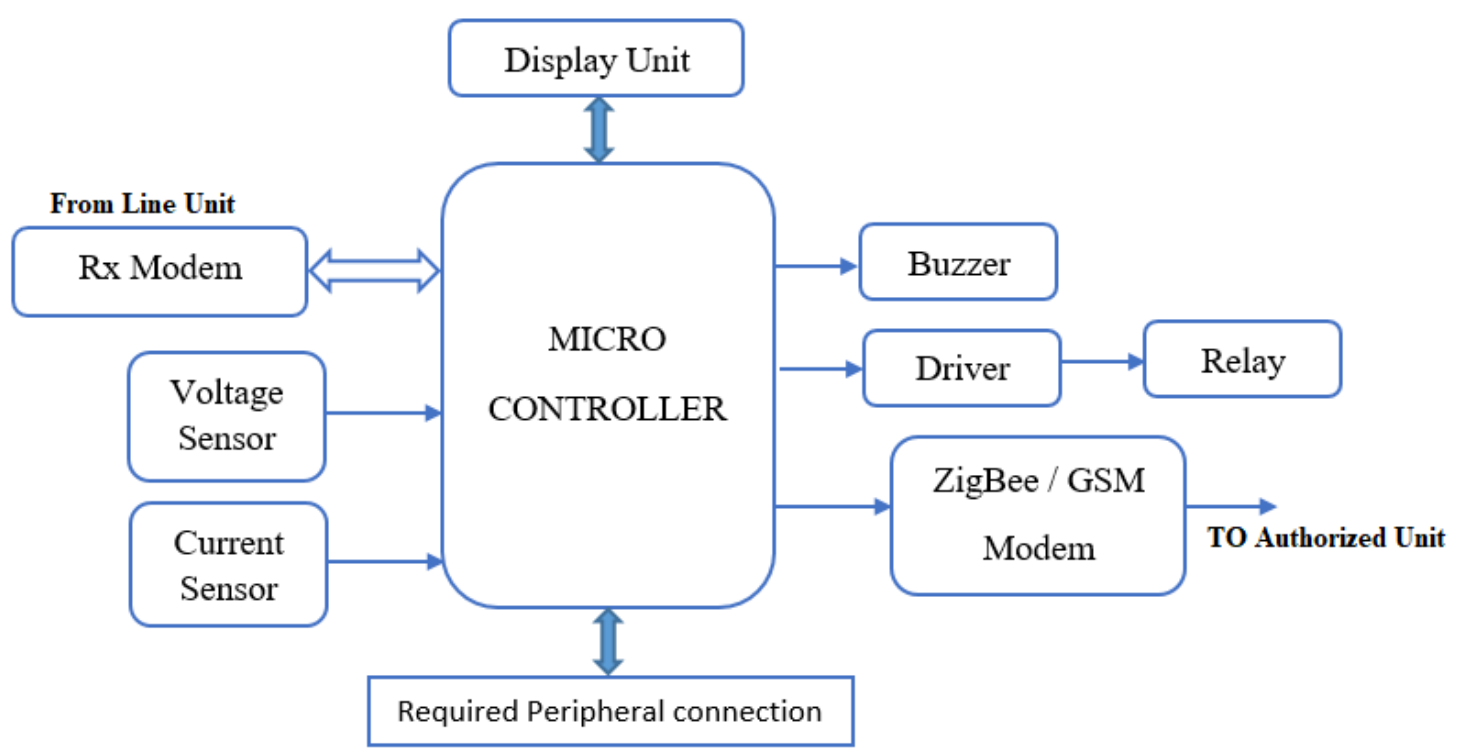

Figure 4 Connected components with microcontroller of master unit 
Journal of Electronics and Informatics (2021)

Vol.03/ No.01

Pages: $36-48$

https://www.irojournals.com/iroei/

DOI: https://doi.org/10.36548/jei.2021.1.004

The master unit of our proposed system comprised many units which is shown in figure 4. The data from the line unit is received by modem to monitor and control the microcontroller unit. The voltage and current values of the line unit can be classified and categorized by the microcontroller to display which pole has failed and the distance is calculated by the microcontroller unit. The master unit is providing a good accurate reading of the line unit of the proposed system through serial port communication between the modem and microcontroller unit. Generally, RS 232 is used for serial communication ports between them. The result of the calculation distance and the faulty pole identification number can be transferred to the authorized person or unit that has an alarm to indicate a faulty status in the transmission line.

\section{RESULTS DISCUSSION}

Table 1 shows performance measures based on the common measuring techniques with an existing system. Every measuring parameter is significant in the computation. The proposed model has higher accuracy in detecting faulty poles in a network. It is concluded that the proposed model identifies much time accurately in the faulty poles. After several iterations, the system's reliability is very excellent.

Table 1 Performance comparison results obtained by Hybrid Model

\begin{tabular}{|c|l|c|c|c|}
\hline S.NO & \multicolumn{1}{|c|}{$\begin{array}{c}\text { MEASURING } \\
\text { METRICS }\end{array}$} & $\begin{array}{c}\text { IMPEDANCE } \\
\text { BASED } \\
\text { METHOD }\end{array}$ & $\begin{array}{c}\text { HYBRID } \\
\text { METHOD }\end{array}$ & $\begin{array}{c}\text { KNOWLEDGE } \\
\text { BASED } \\
\text { METHOD }\end{array}$ \\
\hline 1 & Accuracy & LOW & HIGH & MODERATE \\
\hline 2 & Reliability & LOW & HIGH & MODERATE \\
\hline 3 & Computation Time & HIGH & LOW & OPTIMUM \\
\hline 4 & Authorization Unit & NO & YES & YES \\
\hline 5 & Distance Calculation & NO & YES & YES \\
\hline 6 & Overall Efficiency & LOW & HIGH & MODERATE \\
\hline
\end{tabular}

Our hybrid method is relatively low in computation time compared to existing methods. It is concluding that the system is faster than other system performance. It can be appended with more reliable nodes in its network with new up-gradation. Besides, the authorized units incorporate the hybrid model in order to improve the accuracy and reliability of the system. The 
Journal of Electronics and Informatics (2021)

Vol.03/ No.01

Pages: $36-48$

https://www.irojournals.com/iroei/

DOI: https://doi.org/10.36548/jei.2021.1.004

presence of a distance calculation unit provides a valid impact on our project. Collectively, the overall efficiency is near to very high due to all performance of parameters are relatively high. It can be concluded that the overall performance is excellent by our proposed hybrid efficiency model. Figure 5 shows the overall performance comparison of the proposed hybrid model.

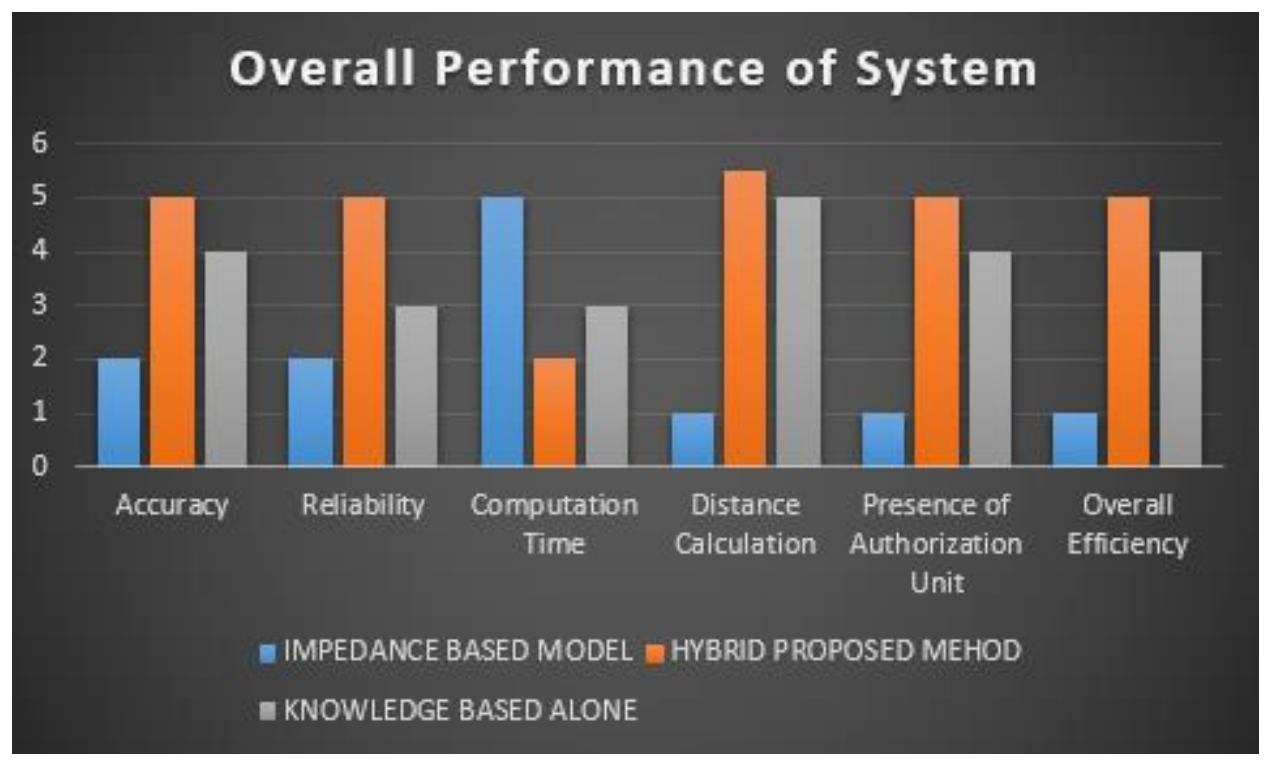

Figure 5 Overall performance comparison of Proposed Model

\section{CONCLUSION}

In this research work, an efficient fault detection technique is developed with the assistance of transmission lines. The causes of overhead power transmission and techniques for identifying them were discussed using existing methods. The knowledge-based method of identification of faulty poles and distance calculation through microcontrollers were discussed. The accuracy and reliability of the proposed system are increased by combining two methods, while the cost expenses are reduced. The proposed system fails in digital conversion much time due to minimum cost integrated circuits. It will be replaced in our extended version of this research work. The computation time of identifying the faulty pole can be increased in further research. Handling high voltage circuits will be implemented in future research. The optimized system will be constructed as our future work. The combination of microcontroller and ZigBee, GSM model is providing good communication protocol for the system. This proposed algorithm 
Journal of Electronics and Informatics (2021)

Vol.03/ No.01

Pages: $36-48$

https://www.irojournals.com/iroei/

DOI: https://doi.org/10.36548/jei.2021.1.004

can be applied for fault detection in the generator and motor. The proposed model will be upgradable to interface many applications to detect the faulty by the human in many remote areas. Besides, the improvement in detection will be taking into industrial automation. It extends to improve the security in the design of the authorization unit of the proposed hybrid model.

\section{REFERENCES}

[1] P.A.Gulbhile, J.R.Rana, B.T.Deshmukh ,Review for Overhead Line Fault Detection Using GSM technology, Vol. 5, Issue 12, December 2016.

[2] Bhanuprakash, Arun. C, Akhil Satheesh . Automatic Power Line Fault Detector. Vol. 6, Special Issue 4, March 2017

[3] Okokpujie Kennedy, Amuta Elizabeth, Okonigene Robert, Samuel John, Monitoring and Fault Detection System For Power Transmission Using Gsm Technology, Int'l Conf. Wireless Network.

[4] K. Nareshkumar, Member, IEEE, M. A. Choudhry, Senior Member, IEEE, J. Lai, A. Feliachi, Senior Member, IEEE. Application of Multi-Agents for Fault Detection and Reconfiguration of Power Distribution Systems. on June 03,2010.

[5] Devanand Samuel S, Bashyam. Fault Detection on Transmission Line Using Thermopile Array and Real time IR Bitmap Generation. 2014 IJEDR | Volume 2, Issue 2 | ISSN: 23219939.Via Bluetooth. Online ISSN: 2249-4596 \& Print ISSN: 0975-5861.

[6] Neeta D. Sonwane, Prof. Devidas D. Dighe . Fault Detection and Autoline Distribution System with GSM Module. Volume 4 Issue IX, September 2016. IJRASETS. Chen, B. Mulgrew, and P. M. Grant, "A clustering technique for digital communications channel equalization using radial basis function networks," IEEE Trans. on Neural Networks, vol. 4, pp. 570-578, July 1993.

[7] Vikramsingh R. Parihar, Line Trap and Artificial Inteligence Based Double Ciruit Tranmission Line Fault Classification, IEEE International Conference on Energy, Communication, Data Analytics and Soft Computing (ICECDS 2017). 
Journal of Electronics and Informatics (2021)

Vol.03/ No.01

Pages: $36-48$

https://www.irojournals.com/iroei/

DOI: https://doi.org/10.36548/jei.2021.1.004

[8] Ing. Komi Agbesi, Felix Attuquaye Okai . AUTOMATIC FAULT DETECTION AND LOCATION IN POWER TRANSMISSION LINES USING GSM TECHNOLOGY. Vol.no.5 issue 01, January 2016

[9] S.Leelakrishnan, V.Ganesharavinth, K.Kalpana, P.Sivaranjani, S.Vijaykumar . Distribution Side Fault Detection and Disconnection Using GSM. Vol. 6, Issue 3, March 2017

[10] Chandra shekar. P .Transmission Line Fault Detection \& Indication through GSM .ISSN (Online): 2347 - 2812, Volume-2, Issue -5, 2014 .

[11] Mr. Nilesh S.Wani, Dr. R. P. Singh . TRANSMISSION LINE FAULTS DETECTION- A REVIEW. Volume 7, Issue 2, March-April, 2016

[12] A. Lavric, V. Popa, C. Males, and I. Finis, "A Performance Study of ZigBee Wireless Sensors Network Topologies for Street Lighting Control Systems,” in 2012 International Conference on Selected Topics in Mobile and Wireless Networking (iCOST), 2012, pp. 130133.

[13] M. N. Jivani, "GSM Based Home Automation System Using App-Inventor for Android Mobile Phone," Int. J. Adv. Res. Electr. Electron. Instrum. Eng., vol. 03, no. 09, pp. 1212112128, 2014.

[14] Xiaoxin $\mathrm{Xu}$, Mingguang $\mathrm{Wu}$, Cheng Ding, Bin Sun, and Jiangwei Zhang, "Outdoor Wireless Healthcare Monitoring System for Hospital Patients based on ZigBee," in 2010 5th IEEE Conference on Industrial Electronics and Applications, 2010, pp. 549- 554.

[15] A. A. Siddiqui, A. W. Ahmad, H. K. Yang, and C. Lee, "ZigBee based Energy Efficient Outdoor Lighting Control System,” Adv. Commun. Technol. (ICACT), 2012 14th Int. Conf., pp. 916-919, 2012.

[16] prof. m. s. sujatha, dr. m vijaykumar. on-line monitoring and analysis of faults in transmission and distribution lines using gsm technique. 30th November 2011. Vol. 33 No.2 () 2005 - 2011 JATIT \& LLS. All rights reserved.

[17] R. N. Patel, Mamta Patel, Fault Detection and Classification on a Transmission Line using Wavelet Multi Resolution Analysis and Neural Network. International Journal of Computer Applications (0975 - 8887) Volume 47- No.22, June 2012

[18] Sushil Chavhan, Vaibhav Barsagade, Abhijit Dutta, Shubhangi Thakre. Fault Detection in Power Line Using Wireless 
Journal of Electronics and Informatics (2021)

Vol.03/ No.01

Pages: $36-48$

https://www.irojournals.com/iroei/

DOI: https://doi.org/10.36548/jei.2021.1.004

[19] By MD Asaduzzaman Nur, Jahidul Islam, Md. Golam Mostofa \& M oshiul Alam Chowdhury Transmission Line Fault Detection Using Android Application

[20] Preethi Manivannan, Prof. Manik Hapse, Fast and Accurate Fault Detection in Transmission Line using Wavelet Transform . Volume 2 | Issue 11 | April 2016. Sensor Network . Volume 3, Issue 3, March 2015. (IIJEE)

[21] Das R., Sachdev, M. S. and Sidhu, T. S. (2000), A Fault Locator for Radial Sub Transmission and Distribution Lines", In Proceedings of IEEE PES Summer Meeting, pp. 443448.

[22] Senger, E. C., Manassero, G., Goldemberg, C. and Pellini, E. L. (2005), Automated Fault Location System for Primary Distribution Networks, IEEE Transactions on Power Delivery, pp. 1332-1340.

[23] Al-Shaher, M., Sabra, M. M. and Saleh, A.S. (2003), Fault Location in Multi-Ring Distribution Network using Artificial Neural Network, Electric Power Systems Research. pp. 8792.

[24] Wang, C., Nouri, H. and Davies, T. S. (2000), A Mathematical Approach for Identification of Fault Sections on the Radial Distribution Systems, 10 Mediterranean Electrotechnical Conference (MELECON), pp. 882-886. 